\title{
MEMRISTIVE BEHAVIOR OF NaOH-IMMERSED TITANIA NANOSTRUCTURES
}

\author{
N. S. Kamarozaman ${ }^{1}$, Z. Aznilinda ${ }^{1}$, R. A. Bakar ${ }^{1}$, S.H. Herman ${ }^{1, *}$ and M. Rusop ${ }^{1}$ \\ ${ }^{1}$ NANO-Electronic CenTre (NET), Faculty of Electrical Engineering, \\ Universiti Teknologi MARA, 40450 Shah Alam, Selangor, Malaysia \\ *Email: hana1617@ salam.uitm.edu.my \\ Phone: +60355436041
}

\begin{abstract}
Memristive behavior is defined as a resistive switching loop which can be observed from the current-voltage (I-V) characteristic of a material. This paper reports the application of $\mathrm{TiO}_{2}$ (titania) nanostructures as an active layer for a memristive device instead of using titania thin film in nanoscale thickness as reported by other researchers. $60 \mathrm{~nm}$ thickness of titania thin film was deposited on ITO-coated glass substrate using the RF-magnetron sputtering method. Then, for the titania nanostructure's growth, the $\mathrm{TiO}_{2} / \mathrm{ITO} /$ glass sample was immersed in $10 \mathrm{~mol} / \mathrm{l}$ aqueous $\mathrm{NaOH}$ solution at $80^{\circ} \mathrm{C}$ while varying the immersion time for 30,45 and 60 min. It was found that the sample immersed for 30 min showed better memristive behavior since larger switching loops were observed when positive bias was applied to the sample. The active layer consists of oxygen-deficient titania where oxygen vacancies might present on the surface of the thin film as the result of $\mathrm{NaOH}$-immersion beside the formation of titania nanostructures. The degradation of the switching loops of the samples immersed in $\mathrm{NaOH}$ solution for 45 and $60 \mathrm{~min}$ might be due to the higher porosity of the samples resulting from the longer immersion process.
\end{abstract}

Keywords: Titania nanostructure; immersion; memristive behavior.

\section{INTRODUCTION}

The theory of a memristor was first theorized by Chua in 1971 (Chua, 1971) and physically realized in 2008 (Williams, 2008), and has attracted much attention from many researchers due to its ability to be used as a nonvolatile memory, switching devices, and smart interconnects (Duraisamy, Muhammad, Kim, Jo, \& Choi, 2012; Haridas, Patil, \& Manjunath, 2010; Yoo, Lee, Tighineanu, \& Schmuki, 2013). The word itself is a short form of "memory resistor" which has the ability to remember its history. As a switching device which has a simpler structure than a transistor, it is a promising candidate to replace transistors for small devices. Any device that exhibits the memristive behavior is called a memristor, the fourth fundamental circuit element which has the relationship between charge and magnetic flux (Hayes, 2011). Many have reported that memristive behavior is only noticeable in devices having nano-scale thickness (Gale, Mayne, Adamatzky, \& Lacy Costello, 2014; Muhammad et al., 2013; Tedesco, Stephey, Hernandez-Mora, Richter, \& Gergel-Hackett, 2012). However, recent study shows that memristive devices can also be fabricated in millimeter scale as well as in microscale thicknesses (Gergel-Hackett et al., 2009; Johnson, Sundararajan, Hunley, \& Strachan, 2010; Prodromakis, Michelakis, \& Toumazou, 2010a, 2010b). The memristive behavior is dependent on the charge displacement within the active 
layer (Miller, Nalwa, Bergerud, Neihart, \& Chaudhary, 2010; Prodromakis, Michelakis, \& Toumazou, 2010c), where the oxygen vacancies control the conductivity and switching behavior of the device. Titania is chosen as a material in fabricating memristors because it exhibits good memristive behavior (Aznilinda, Herman, Bakar, \& Rusop, 2013; Kamarozaman et al., 2013; Li et al., 2010; Miller et al., 2010). Yanagida (2011) have successfully fabricated memristive devices using single oxide nanowires $\left(\mathrm{CoO}_{\mathrm{x}}, \mathrm{NiO}, \mathrm{TiO}_{2}\right)$ by the photolithography method, and Haykel Ben Jamaa (2009) show that memristors can also be fabricated based on poly-crystalline silicon nanowires by Low Pressure Chemical-Vapor-Deposition (LPCVD). Both papers proved that nanostructured materials can be applied as an active layer for memristive devices which exhibit excellent performance.

Great efforts have been made in synthesizing one-dimensional (1-D) $\mathrm{TiO}_{2}$ materials due to their unique and excellent properties in wide applications such as in optics, electronics, solar cells and photocatalysts (Kumar, Madaria, \& Zhou, 2010). Most have reported growing $\mathrm{TiO}_{2}$ nanostructures using $\mathrm{NaOH}$ solution by a hydrothermal method where a high temperature is required for the process (Shao, Sun, Gao, Yang, \& Luo, 2011; Xie, Wang, \& Zhou, 2012; Zhu et al., 2005). However, in this work, we attempt to grow $\mathrm{TiO}_{2}$ nanostructures at low temperature. By contrast with the conventional method of growing nanostructured materials where metals such as $\mathrm{Au}$ and $\mathrm{Al}$ are being used as the catalyst, in this work, we used a $\mathrm{TiO}_{2}$ thin film as the nanostructure growth seed layer. This is to overcome the leakage current problem caused by the remnant metallic material even after removal of the metal catalyst. The effect of the $\mathrm{NaOH}$ immersion time on the nanostructure morphology and electrical properties of the $\mathrm{TiO}_{2}$ nanostructures for memristive behavior was studied.

\section{METHODOLOGY}

ITO-coated glass substrate (ITO: $200 \mathrm{~nm}$ thickness as a bottom electrode) was cleaned using a standard cleaning method with acetone and methanol followed by distilled water. Then the substrate was dried using nitrogen gas. $60 \mathrm{~nm}-\mathrm{TiO}_{2}$ thin film was deposited on the conducting side of the ITO substrate using $\mathrm{TiO}_{2}(99.999 \%$ purity) as a target by the RF magnetron sputtering method for $5 \mathrm{~min}$, at $300 \mathrm{~W} \mathrm{RF}$ power and a working pressure of 5 mTorr. During the deposition process, $50 \mathrm{sccm}$ of argon gas was introduced into the chamber with the substrate heated at $200{ }^{\circ} \mathrm{C}$. $\mathrm{TiO}_{2}$ nanostructures were grown on the $\mathrm{TiO}_{2}$ thin film through an immersion method by using $10 \mathrm{~mol} / \mathrm{l}$ aqueous $\mathrm{NaOH}$ solution at $80^{\circ} \mathrm{C}$ with the setup shown in Figure $1 . \mathrm{TiO}_{2}$ thin film as a seed layer for the nanostructures growth was immersed with the $\mathrm{TiO}_{2}$ layer facing down in the vessel for 30, 45 and $60 \mathrm{~min}$. Then, the sample was taken out of the vessel and rinsed several times with $0.1 \mathrm{M}$ nitric acid to remove the sodium ions. The sample was annealed at $450^{\circ} \mathrm{C}$ for 1 hour to enhance the crystallinity of the nano-featured $\mathrm{TiO}_{2}$ layer. Pt as a top electrode for the device was sputtered with $60 \mathrm{~nm}$ thickness. The device structure in this work was then $\mathrm{Pt} / \mathrm{TiO}_{2}$ nanostructures/ $/ \mathrm{TiO}_{2} / \mathrm{ITO} /$ glass. The current-voltage measurement was performed at room temperature by the two-point probe method using a Keithley 4200 semiconductor characterization system connected to a probe station. To test the memristive behavior, the bias voltage is swept from $0 \mathrm{~V}$ to $5 \mathrm{~V}$, then $5 \mathrm{~V}$ to $-5 \mathrm{~V}$ and back to $0 \mathrm{~V}$ while simultaneously measuring the current. The surface morphology and cross-section image of the thin films was measured using a field emission scanning electron microscope (FESEM, JEOL JSM 7600F). The 
composition of the thin film was measured with energy dispersive x-ray spectroscopy (EDS).

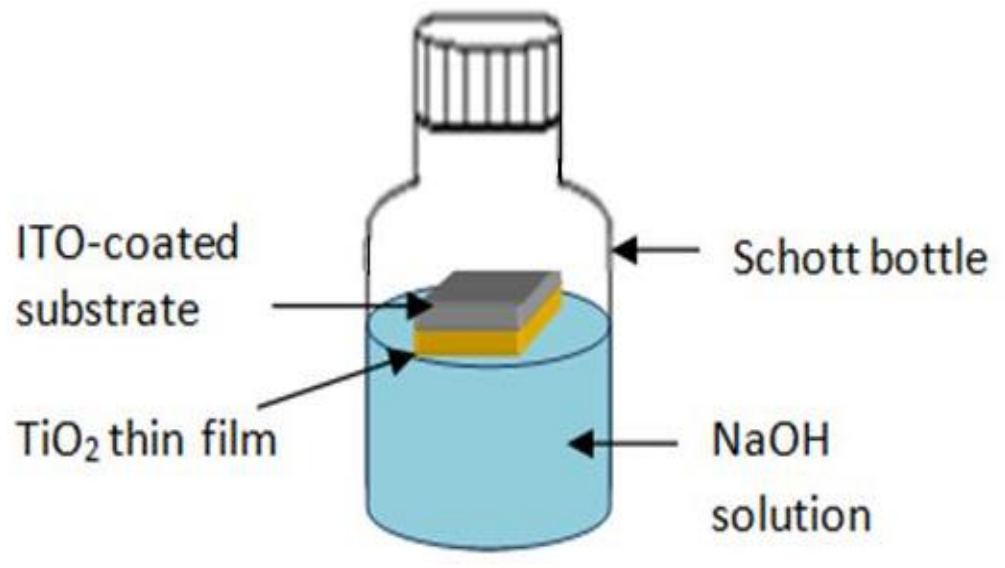

Figure 1. Schematic diagram for the sample immersed in $\mathrm{NaOH}$ solution (Kamarozaman et al., 2013)

\section{RESULTS AND DISCUSSION}

The cross-sections of the samples immersed in $\mathrm{NaOH}$ solution for 30, 45 and 60 min are shown in Figure 2. The insets show the corresponding surface morphology images of the samples. The cross-sectional image for the as-deposited sample in Figure 2 (a) shows that the grains are denser. The inset image shows the formation of $\mathrm{TiO}_{2}$ thin film with agglomerated $\mathrm{TiO}_{2}$ nanoparticles. As reported by Xu and Wang (2011), when the substrate is placed floating on the solution as shown in Figure 1, this will prevent any precipitates from the bulk solution falling onto the substrate, which would otherwise inhibit the growth of the desired nanostructures and possibly initiate secondary growth. Figure 2 (b) shows the formation of $\mathrm{TiO}_{2}$ nanomesh at an early stage via the reaction between native titanium oxides and $\mathrm{NaOH}$ solution after being immersed in $\mathrm{NaOH}$ solution for $30 \mathrm{~min}$. The cross-sectional image shows that $\mathrm{TiO}_{2}$ nanostructures grew on the pre-existing $\mathrm{TiO}_{2}$ thin film. Further extending the $\mathrm{NaOH}$ immersion time to 45 and 60 min results in dandelion-like morphologies of $\mathrm{TiO}_{2}$ nanostructures, as shown in Figure 2 (c) and (d). It can be said that the etching and growth mechanism of $\mathrm{TiO}_{2}$ nanostructures occur at the same time due to the longer immersion time. Further explanation of how this nucleation of nanostructure growth occurs is illustrated in Figure 3. When the substrate was immersed into concentrated $\mathrm{NaOH}$ solution, the surface of the film was being etched away. As the dissolution continues, more elemental Ti from the bulk $\mathrm{TiO}_{2}$ thin film dissolves into the solution and may lead to a rough surface. However, these dissolved atoms may be either trapped on the rough surface, forming nucleation sites for further $\mathrm{TiO}_{2}$ nanostructure growth (Kumar et al., 2010; Xie et al., 2012) or re-adsorbed. The etching rate may be faster than the nanostructure growth rate, resulting in higher porosity of the samples in longer immersion processes. This growth mechanism is illustrated in Figure 3. 

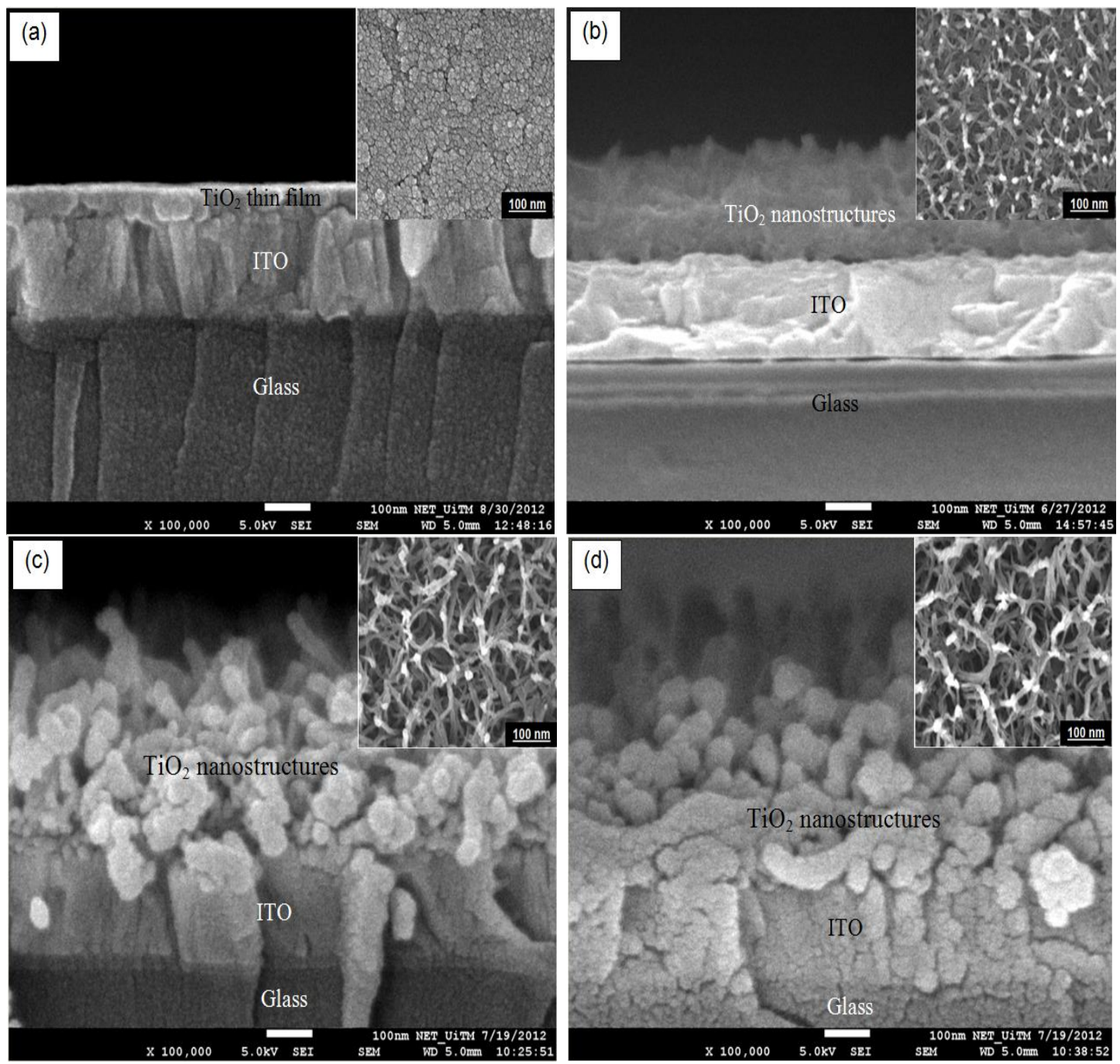

Figure 2. Surface morphology of the (a) as-deposited sample, and immersed in $\mathrm{NaOH}$ solution for (b) $30 \mathrm{~min}$, (c) $45 \mathrm{~min}$, and (d) $60 \mathrm{~min}$.

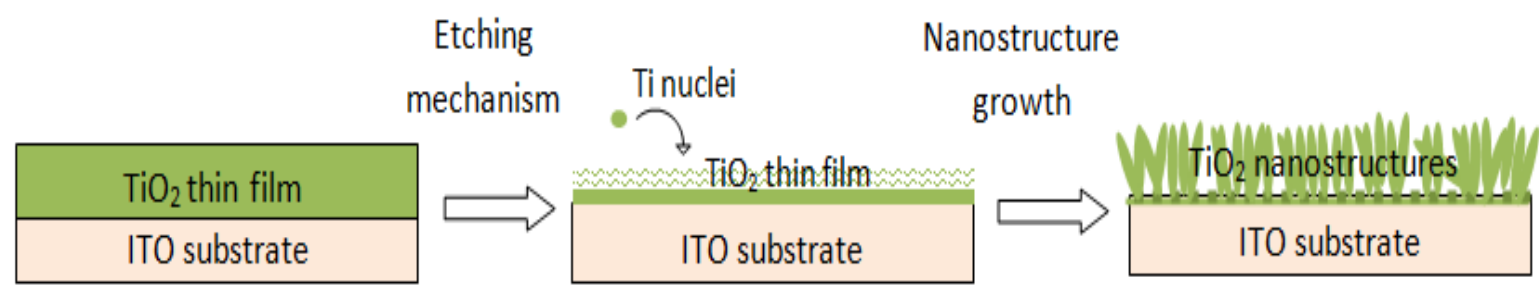

Figure 3. Schematic diagram of growth mechanism of $\mathrm{TiO}_{2}$ nanostructures on $\mathrm{TiO}_{2}$ thin film.

Figures 4 (a) to (d) show the current-voltage measurements for the as-deposited sample and the samples immersed in $\mathrm{NaOH}$ solution for 30, 45 and $60 \mathrm{~min}$. Figure 4 (a) shows an ohmic I-V curve for the as-deposited $\mathrm{TiO}_{2}$ thin film sample. Meanwhile, Figures 4 (b) to (d) show the switching characteristics of $\mathrm{TiO}_{2}$ nanostructures as an active layer for the memristive device. The samples in Figures 4 (b) and (c) show switching loops when positive voltage is applied to the samples, probably due to the oxygen vacancies present within the $\mathrm{TiO}_{2}$ layer. 

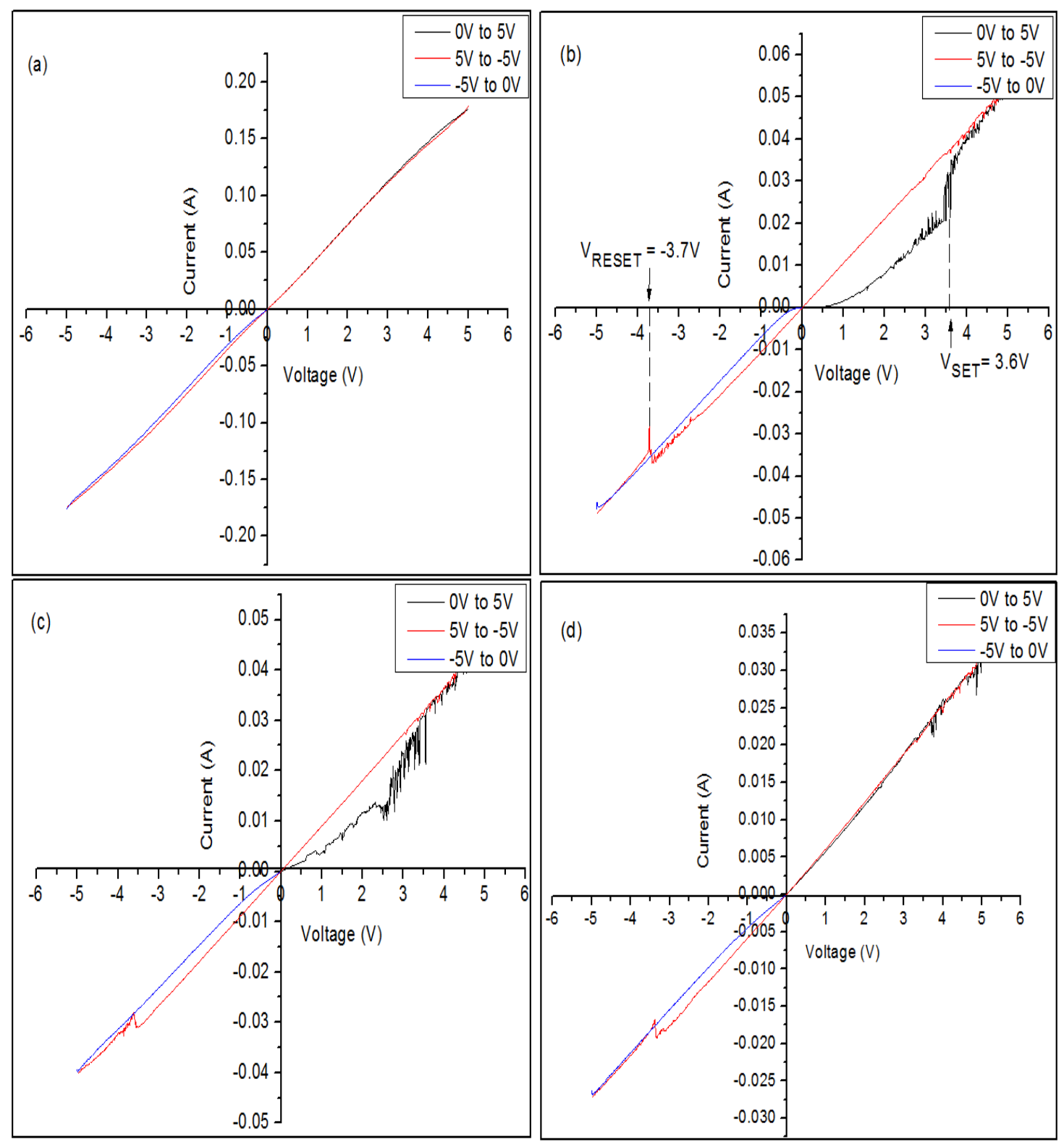

Figure 4. Current-voltage measurement for memristive device of (a) as-deposited sample, and immersed in $\mathrm{NaOH}$ solution at (b) $30 \mathrm{~min}$, (c) $45 \mathrm{~min}$, and (d) $60 \mathrm{~min}$.

It was observed at $5 \mathrm{~V}$ that the maximum current of the sample immersed for 30 min (Figure 4 (b)) is 0.06A, while the samples immersed for 45 (Figure 4 (c)) and 60 min (Figure 4 (d)) give maximum current of $0.05 \mathrm{~A}$ and $0.035 \mathrm{~A}$. Thus, this in return suggests that the graph shows an insulative I-V curve as the $\mathrm{NaOH}$ immersion time increases to $60 \mathrm{~min}$. The higher conductivity of the I-V curve is believed to be due to the higher ion concentrations of positively charged oxygen vacancies within the $\mathrm{TiO}_{2}$ layer (Williams, 2008). This is supported by the EDS measurement taken on the surface of the $\mathrm{TiO}_{2}$ layer, as shown in Table 1 below. The sample in Figure 4 (b) is assumed to switch from the off-state (high resistance state (HRS)) to the on-state (low resistance state (LRS)) at $\mathrm{V}=3.6 \mathrm{~V}$, and from the on-state (LRS) to the off-state (HRS) at V=-3.7 V. Figure 4 (c) shows switching loops at positive sides, but the difference in magnitude between the two states is difficult to see. Meanwhile, Figure 4 (d) shows no hysteresis 
loop at the positive sides, and only a slight hysteresis loop was observed at the negative sides. The switching loops start to degrade for 45 and $60 \mathrm{~min}$ when positive voltage is applied to the sample, which might be due to the increase of porosity within the $\mathrm{TiO}_{2}$ layer. This porous layer increases the grain boundaries between the $\mathrm{TiO}_{2}$ particles, thus slowing down the electron movement, which then affects the performance of the memristive behavior.

The oxygen concentration of the as-deposited sample and the samples immersed for 30, 45 and 60 min in $\mathrm{NaOH}$ solution was analyzed using EDS measurements. The results indicated that the oxygen concentration within the $\mathrm{TiO}_{2}$ layer decreased with $\mathrm{NaOH}$ immersion time. The longer the surface of $\mathrm{TiO}_{2}$ thin film was immersed in $\mathrm{NaOH}$ solution, the more porosity occurred, along with the removal of oxygen caused by the etching process, resulting in more oxygen vacancies within the $\mathrm{TiO}_{2}$ layer. Thus, the samples immersed in $\mathrm{NaOH}$ solutions should give better memristive behavior than the as-deposited sample. However, although higher oxygen vacancies are more favorable for better memristive behavior, longer $\mathrm{NaOH}$ immersion time results in the porosity of the $\mathrm{TiO}_{2}$ film, which might degrade the performance of the memristive behavior.

Table 1. Oxygen concentration of the $\mathrm{TiO}_{2}$ films with $\mathrm{NaOH}$ immersion time.

\begin{tabular}{ccc}
\hline Sample & NaOH immersion time (minutes) & Oxygen concentration (wt\%) \\
\hline A & As-deposited & 42.81 \\
$\mathrm{~B}$ & 30 & 29.58 \\
$\mathrm{C}$ & 45 & 27.49 \\
$\mathrm{D}$ & 60 & 27.24 \\
\hline
\end{tabular}

\section{CONCLUSIONS}

In this work, $\mathrm{TiO}_{2}$ dandelion-like nanostructures were synthesized by a simple immersion method using $10 \mathrm{M} \mathrm{NaOH}$ solution. The sputtered titania thin film was immersed in $\mathrm{NaOH}$ solution while varying the $\mathrm{NaOH}$ immersion time from 30, 45 and 60 min. The effect of $\mathrm{NaOH}$ immersion time on the nanostructure morphology and its memristive behavior was studied. Thus, the device structure in this work is a $\mathrm{Pt} / \mathrm{TiO}_{2}$ nanostructures/ $/ \mathrm{TiO}_{2} / \mathrm{ITO}$ memristive device. From the result, $\mathrm{TiO}_{2}$ nanostructures exhibited better memristive behavior than bulk $\mathrm{TiO}_{2}$ thin film. The sample immersed in $\mathrm{NaOH}$ solution for 30 min shows larger switching loops when positive voltage is applied to the sample, probably due to the existence of extra oxygen vacancies induced by the $\mathrm{NaOH}$ etching process. However, samples immersed for 45 and 60 min show degradation of the switching loops when positive voltage is applied to the sample, which might be due to the higher porosity of the samples in the longer immersion process.

\section{ACKNOWLEDGEMENTS}

This work is partially supported by the Research Management Institute (RMI), UiTM and Malaysian Ministry of Higher Education (MOHE) under the Fundamental Research Grant Scheme (FRGS/1/ 2012/ TK02/ UITM /03/8) and E-Science (Project code : 100RMI/SF 16/6/2 (31/2012)). 


\section{REFERENCES}

Aznilinda, Z., Herman, S. H., Bakar, R. A., \& Rusop, M. (2013). Effect of electrode types on the resistive switching behavior of titania thin films. Applied Mechanics and Materials, 393, 74-78.

Chua, L. (1971). Memristor - the missing circuit element. Paper presented at the IEEE Transactions on Circuit Theory.

Duraisamy, N., Muhammad, N. M., Kim, H. C., Jo, J. D., \& Choi, K. H. (2012). Fabrication of tio2 thin film memristor device using electrohydrodynamic inkjet printing. Thin Solid Films, 520, 5070-5074.

Gale, E., Mayne, R., Adamatzky, A., \& Lacy Costello, B. (2014). Drop-coated titanium dioxide memristors. Materials Chemistry and Physics, 143, 524-529.

Gergel-Hackett, N., Hamadani, B., Dunlap, B., Suehle, J., Richter, C., Hacker, C., \& Gundlach, D. (2009). A flexible solution-processed memristor. IEEE Electron Device Letters, 30, 706-708.

Haridas, M., Patil, S., \& Manjunath, T. C. (2010). Recent atc's in the design of memristors. International Journal of Computer Electrical Engineering, 2, 17938163.

Hayes, B. (2011). The memristor. American Scientist, 99, 106-110.

Haykel Ben Jamaa, M. e. a. (2009). Fabrication of memristors with poly-crystalline silicon nanowires. Paper presented at the 9th IEEE Conference on Nanotechnology.

Johnson, S. L., Sundararajan, A., Hunley, D. P., \& Strachan, D. R. (2010). Memristive switching of single-component metallic nanowires. Nanotechnology, 21, 125204.

Kamarozaman, N. S., Asiah, M. N., Aznilinda, Z., Bakar, R. A., Abdullah, W. F. H., Herman, S. H., \& Rusop, M. (2013). Memristive behavior of tio2 nanostructures grown at different substrate positioning by immersion method. Advanced Materials Research, 795, 256-259.

Kumar, A., Madaria, A. R., \& Zhou, C. (2010). Growth of aligned single-crystalline rutile tio2 nanowires on arbitrary substrates and their application in dyesensitized solar cells. Journal of Physical Chemistry C, 14, 7787-7792.

Li, Y. T., Long, S. B., Lu, H. B., Liu, Q., Wang, Q., Wang, Y.et al.Liu, M. (2010). A low-cost memristor based on titanium oxide. Paper presented at the 10th IEEE International Conference on Solid-State and Integrated Circuit Technology (ICSICT).

Miller, K., Nalwa, K. S., Bergerud, A., Neihart, N. M., \& Chaudhary, S. (2010). Memristive behavior in thin anodic titania. Paper presented at the IEEE Electron Device Letters.

Muhammad, N. M., Duraisamy, N., Rahman, K., Dang, H. W., Jo, J., \& Choi, K. H. (2013). Fabrication of printed memory device having zinc-oxide active nanolayer and investigation of resistive switching. Current Applied Physics, 13, 9096.

Prodromakis, T., Michelakis, K., \& Toumazou, C. (2010a). Switching mechanisms in microscale memristors. IEEE Electron Device Letters, 46, 63-65.

Prodromakis, T., Michelakis, K., \& Toumazou, C. (2010b). Practical micro/nano fabrication implementations of memristive device. Paper presented at the 12th 
International Workshop on Cellular Nanoscale Networks and Their Applications (CNNA).

Prodromakis, T., Michelakis, K., \& Toumazou, C. (2010c). Fabrication and electrical characteristics of memristors with tio2/tio $2+x$ active layers. Paper presented at the Proc. of 2010 IEEE International Symposium on Circuits and Systems (ISCAS).

Shao, F., Sun, J., Gao, L., Yang, S. W., \& Luo, J. Q. (2011). Template-free synthesis of hierarchical tio2 structures and their application in dye-sensitized solar cells. ACS Applied Materials \& Interfaces, 3, 2148-2153.

Tedesco, J. L., Stephey, L., Hernandez-Mora, M., Richter, C. A., \& Gergel-Hackett, N. (2012). Switching mechanisms in flexible solution-processed tio2 memristors. Nanotechnology, 23.

Williams, R. (2008). How we found the missing memristor. IEEE Spectrum, 45, 28-35.

Xie, J., Wang, X., \& Zhou, Y. (2012). Understanding formation mechanism of titanate nanowires through hydrothermal treatment of various ti-containing precursors in basic solutions. Journal of Materials Science \& Technology, 28(6), 488-494.

Xu, S., \& Wang, Z. L. (2011). One-dimensional zno nanostructures: Solution growth and functional properties. Nano Research, 4, 1013-1098.

Yanagida, T. (2011). Memristive switching phenomena in a single oxide nanowire. Paper presented at the IEEE Nanotechnology Materials and Devices Conference.

Yoo, J. E., Lee, K. Y., Tighineanu, A., \& Schmuki, P. (2013). Highly ordered tio2 nanotube-stumps with memristive response. Electrochemistry Communications, 34, 177-180.

Zhu, H. Y., Lan, Y., Gao, X. P., Ringer, S. P., Zheng, Z. F., Song, D. Y., \& Zhao, J. C. (2005). Phase transition between nanostructures of titanate and titanium dioxides via simple wet-chemical reactions. Journal of the American Chemical Society, $127,6730-6736$. 